UNIVERSITE DE LAUSANNE-FACULTE DE BIOLOGIE ET DE MEDECINE

DEPARTEMENT DE PSYCHIATRIE

CENTRE DE NEUROSCIENCES PSYCHIATRIQUES

CHEF DE SERVICE : PROFESSEUR Pierre MAGISTRETTI

\title{
Recommandations de pratique clinique dans la schizophrénie : de la théorie à la mise en application
}

\section{THESE}

\section{Préparée sous la direction du}

Professeur Pierre Baumann

et présentée à la

Faculté de Biologie et de Médecine de l'Université de Lausanne pour l'obtention du grade de

DOCTEUR EN MEDECINE

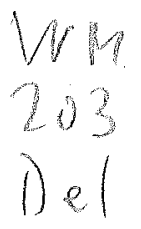

par PMTE 3456

\section{Didier Delessert}

Médecin diplômé de la Confédération Suisse

Originaire de Cully-Lutry (VD) 


\section{Résumé}

Dans le cadre d'une étude rétrospective au sein d'une unité de réhabilitation, nous avons cherché à examiner le degré de respect de recommandations de pratique clinique (RPC) abordant le traitement pharmacologique au long cours de la schizophrénie, par dẹs médecins qui n'en ont qu'une connaissance indirecte.

"The Expert Consensus Guideline for the treatment of schizophrenia » (« ECGTS ») a été retenu comme référence sur la base d'une comparaison avec cinq autres RPC principales.

Sur un collectif de 20 patients, les recommandations de «l'ECGTS» sont totalement respectées dans $65 \%$ des cas, partiellement respectées dans $10 \%$ et non respectées dans $25 \%$, démontrant ainsi que la pratique clinique est clairement perfectible (principalement dans le traitement des symptômes psychotiques et dépressifs). Cependant, le respect des RPC ne garantit pas forcément la résolution de tous les problèmes cliniques rencontrés : 12 patients sur 20 présentent des effets secondaires à l'évaluation clinique, et pour 8 d'entre eux, les recommandations à ce niveau sont respectées.

Notre étude montre cependant que le choix et l'application d'une RPC n'est pas simple. Les RPC actuelles donnent peu ou pas d'instrument de mesure, ni de critère précis pour évaluer les problèmes cliniques auxquels elles font référence. L'avenir appartient donc à des RPC qui proposent, outre les recommandations cliniques elles-mêmes, les moyens de leur vérification et de leur application sur le terrain.

mots clés: schizophrénie, recommandations de pratique clinique, pharmacothérapie, "The Expert Consensus Guideline for the treatment of schizophrenia", réhabilitation, application 


\title{
RECOMMANDATIONS DE PRATIQUE CLINIQUE DANS LA
}

\section{SCHIZOPHRENIE : de la théorie à la mise en application}

\author{
Didier Delessert ${ }^{1}$ \\ Valentino Pomini ${ }^{2}$ \\ François Grasset ${ }^{2}$ \\ Pierre Baumann ${ }^{3}$
}

1.

Service de Médecine et Psychiatrie Pénitentiaires

Département de Psychiatrie-CHUV

Université de Lausanne

Site de Cery, 1008 Prilly.

Suisse

2.

Unité de réhabilitation

Département de Psychiatrie-CHUV

Université de Lausanne

Site de Cery, 1008 Prilly.

Suisse

3.

Unité de Biochimie et de Psychopharmacologie Clinique

Centre de neurosciences psychiatriques

Département de Psychiatrie-CHUV

Université de Lausanne

Site de Cery, 1008 Prilly.

Suisse

Correspondance:

Didier Delessert

Service de Médecine et Psychiatrie Pénitentiaires

Département de Psychiatrie-CHUV

Université de Lausanne

Site de Cery, 1008 Prilly.

Suisse

Tél : 0041216436860 


\section{"Clinical Practice Guidelines for Schizophrenia Treatment : theory to the practice"}

\section{Summary}

Based on the concept of evidence based medicine, the Clinical Practice Guidelines (CPG) occupy an increasingly important place in the field of the treatment of schizophrenia. If there is a CPG elaborated in a rigorous way, it should be noted that few of them provide the readers tools for their operationnalisation. The clinician must then create his own method of application. This problem was encountered within the framework of a retrospective study carried out in a rehabilitation unit, where we sought to answer the question concerning the use of CPG approaching the pharmacological treatment of schizophrenia: "What is the degree of respect of these CPG by doctors who have only an indirect knowledge?"

The comparative study between CPG and practical current clinic implied: 1) the choice of a pertinent CPG for the clinical framework studied, 2) the selection of measure instruments, which allow quantifying obvious clinical problems approached in the CPG, 3) the development of a standardized system of comparison to determine the degree of respect of the recommendations. "The Expert Consensus Guideline for the treatment of schizophrenia" ("ECGTS") was selected as reference. A method of application of the ECGTS is depicted: use of standardized clinical scales ; translation of the results of the clinical examination in terms of clinical problems to which recommendations of the guideline refers; and determination of the degree of respect of the recommendations.

In the group of 20 patients included in this study, the recommendations of the ECGTS are totally respected in $65 \%$ of the patients, and partially respected in $10 \%$ of the subjects, while in $25 \%$ of the patients, they are not respected. These observations suggest that the clinical approach has to be improved, mainly with regard to the treatment of psychotic and depressive symptoms. This work also showed the limits of the CPG: for example, over half of the patients presented side effects at the clinical evaluation whereas the regulation of their medication respects the recommendations of the "ECGTS".

The future certainly belongs to CPG, which proposes, in addition to the clinical recommendations themselves, a method to check their application in clinical practice.

Keywords: schizophrenia, clinical practice guidelines, pharmacotherapy, "Expert Consensus Guideline for the treatment of schizophrenia", rehabilitation, application, recommendations 


\section{Introduction}

Le concept de médecine fondée sur des preuves ou «Evidence-Based Medicine (EBM) 》 s'est développé ces dix dernières années avec comme corollaire l'élaboration d'outils, telles que les recommandations pour la pratique clinique $(\mathrm{RPC}){ }^{1}$, afin de trier et synthétiser l'abondante littérature médicale. Les RPC offrent cependant une qualité ou une présentation variables $(6,10)$, posant la question du choix d'utiliser une RPC plutôt qu'une autre. Plusieurs auteurs ont ainsi proposé des critères pour l'évaluation de la qualité des $\operatorname{RPC}(5,18)$.

Parmi les RPC concernant le traitement de la schizophrénie, très peu fournissent des outils permettant de les opérationnaliser, obligeant le clinicien à créer sa propre méthode d'application (qui aura notamment le défaut de ne pas être standardisée).

L'étude rétrospective que nous avons menée au sein d'une unité de réhabilitation psychiatrique dans le but d'examiner le degré de respect de recommandations de pratique clinique (RPC) abordant le traitement pharmacologique au long cours de la schizophrénie (par des médecins qui n'ont qu'une connaissance indirecte de ces RPC) met en lumière un certain nombre de dífficultés rencontrées par le clinicien désireux d'utiliser celles-ci.

\section{Méthode}

Pour mener notre étude comparative entre RPC et pratique clinique courante nous avons adopté la démarche suivante :

1) le choix d'une RPC pertinente pour le cadre clinique étudié,

2) la sélection d'instruments de mesure permettant d'opérationnaliser les problèmes cliniques abordés dans la RPC,

3) la mise au point d'un système standardisé de comparaison pour déterminer le degré de respect des recommandations.

4) passation des différents instruments par les patients de l'unité et étude de leurs dossiers pour évaluer la manière dont les recommandations sont respectées.

\footnotetext{
${ }^{l_{1}}$ Les termes anglais « clinical practice guidelines " ou " guidelines » ont été traduits dans cet article par « recommandations pour la pratique clinique » (RPC). Le terme « recommandation » est utilisé pour désigner une recommandation particulière dans une RPC.
} 


\section{1) choix de la RPC}

Les six principales RPC abordant le traitement de la schizophrénie $(2,3,13,14,16,17)$, présentées dans le tableau I, ont été répertoriées et évaluées (Delessert et col., soumis pour publication) sur la base de plusieurs critères de qualité dont $(5,18)$ : la date de publication ou période de revue de littérature concernée, la validité scientifique, la facilité d'emploi, le nombre de recommandations concernant la pharmacothérapie.

\section{Insérer Tableaul}

"The Expert Consensus Guideline for the treatment of schizophrenia » (16) («ECGTS 》) a été sélectionné. Cette RPC nous est apparue très complète, apportant le plus de réponses aux préoccupations pharmacothérapeutiques des cliniciens, et la plus pertinente pour une population de patients chroniques. Sa validité scientifique moyenne est directement liée au fait qu'elle aborde plus de problèmes cliniques que les autres. En effet, elle fournit des recommandations généralement contenues dans les autres RPC, mais apporte également une aide dans des domaines où il existe encore peu d'évidence clinique et où les autres RPC n'explicitent pas de recommandations consensuelles (effets secondaires, comorbidités, etc.). Son spectre plus large compense en quelque sorte sa validité scientifique inférieure à celle de «l'APA Practice guidelines » et son guide rapide de réréfence $(13,3)$ ou celle des recommandations « PORT » (14) (cf. Tableau I).

"L'ECGTS (16) est composé de 19 RPC principales, abordant plusieurs niveaux de la prise en charge (pharmacothérapie, interventions psychosociales, etc.) et répartis dans 4 grands chapitres :

I. Stratégies pour la sélection des traitements pharmacologiques; II. stratégies pour la sélection des services de soins; III. questions concernant l'évaluation des patients; IV. questions de politique de santé. L'étude ne portant que sur la pharmacothérapie, seuls les problèmes cliniques abordés dans chapitre I de «l'ECGTS » (16) ont été considérés. Ils sont présentés dans le tableau II.

\section{Insérer Tableau II}

\section{2) Instruments de mesure utilisés pour évaluer les éventuels problèmes cliniques}

L'examen clinique quantitatif a porté sur: 
a) la psychopathologie ("Positive and Negative Syndrome Scale [PANSS]", "Calgary depression scale $»)(1,7,11,12)$,

b) l'identification d'effets secondaires de la médication (L'échelle d'évaluation « UKU » des effets secondaires [UKU], "Abnormal involuntary movement scale [AIMS] », « Barnes akathisia rating scale », «Simpson Angus scale for extrapyramidal side effects ») $(4,8,9,15,21)$.

II a été complété par des informations obtenues auprès des thérapeutes. Les données sociodémographiques, le(s) diagnostic(s) psychiatrique(s), le(s) diagnostic(s) somatique(s) et la médication prescrite au moment de l'examen d'un patient donné et dans les trois mois précédents ont été relevés sur la base des informations contenues dans le dossier médical.

\section{3) Evaluation du respect des recommandations}

La mise en évidence d'un symptôme ou d'un effet secondaire ne justifie pas forcément un changement ou une adaptation de la médication. II faut tenir compte de leur gravité et de leur répercussion dans la vie du patient ou pour sa santé. Ainsi, pour évaluer la gravité de ces répercussions avec les échelles "PANSS », "UKU.», "Barnes akathisia rating scale », nous avons établi arbitrairement des seuils qui définissent la présence d'un problème clinique (correspondant à un effet secondaire ou un symptôme suffisamment grave, justifiant ainsi l'application des RPC de «l'ECGTS 》). Concernant les 3 autres échelles, nous nous sommes référés à la littérature et aux valeurs seuils proposées par différents autẹrs pour évaluer la dépression, un éventuel syndrome extra-pyramidal ou d'autres mouvements anormaux $(1,12,15,21)$. Le tableau III résume les valeurs seuils des échelles et décrit la correspondance entre les RPC de «l'ECGTS » et les problèmes cliniques mis en évidence par ces dernières.

Insérer Tableau III

Dans un second temps, la médication prescrite a été comparée avec celle proposée par les RPC principales correspondantes du chapitre I de «l'ECGTS » (16).

Les RPC 2 à 7 sont constituées de plusieurs recommandations spécifiques dont le degré de respect a été évalué séparément à l'aide d'une échelle en trois points : non-respect (0 point), respect partiel (1 point), respect total (2 points). Un score moyen par RPC a ensuite été calculé sur la base des scores obtenus pour les recommandation spécifiques. Ce score moyen indique le degré de respect de 
la RPC envisagée : un score < ou = à 0,5 signale un «non-respect »; un score situé entre 0,5 et 1,5 marque « un respect partiel », tandis qu'un score > à 1,5 dénote un « respect total » de la RPC.

La RPC $8 \mathrm{~A}$ a dû être traitée différemment des autres. Nous avons attribué une note de +1 aux arguments en faveur d'un changement de traitement antipsychotique et une note de -1 à ceux en faveur d'une absence de changement. Une somme des scores $>0$ indique la nécessité d'un changement de traitement et par-là même un non-respect de cette huitième RPC. Une somme $<$ ou $=$ à 0 témoigne ainsi du respect de la RPC.

Les RPC 1 (traitement initial pour un épisode aigü) et 8B (procédé de modification du traitement neuroleptique) n'ont pas été considérées dans l'étude: la RPC 1 est non pertinente pour une population de patients chroniques et la nature rétrospective de l'étude, avec une seule mesure dans le temps, ne permet pas une analyse précise du degré d'application des recommandations de la RPC $8 B$.

Lorsqu'une RPC dans son ensemble ou une recommandation spécifique qui la compose n'était pas pertinente pour la situation clinique donnée, aucun score n'a été attribué.

\section{Résultats:}

\section{a) Echantillon}

20 sujets ( 2 femmes et 18 hommes, avec une moyenne d'âge de 37 ans [écart-type : 11,5] )

souffrant d'un trouble appartenant au spectre de la schizophrénie, selon CIM-10 (19), ont participé à l'étude : schizophrénie paranoïde ( 6 sujets), schizophrénie hébéphrénique (4 sujets), schizophrénie indifférenciée (4 sujets), schizophrénie résiduelle (1 sujet), trouble schizotypique ( 3 sujets) et trouble schizo-affectif (2 sujets). 11 patients présentent une comorbidité psychiatrique.

Les scores obtenus aux sous-échelles de la «PANSS » $(7,11)$ montrent que notre échantillon se compose de patients relativement stabilisés sur le plan de la symptomatologie positive mais davantage marqués par un syndrome négatif. Comparés aux normes établies par les auteurs de la «PANSS » $(7,11)$, les scores obtenus à l'échelle positive pour 13 sujets se situent en dessous du 
centile 20 (score «PANSS»<15), 6 sujets se situent entre les centiles 20 et 50 (score < 20) et un seul sujet présente une symptomatologie positive très marquée (score $=27$; centile 86 ). Pour la symptomatologie négative, 11 sujets sont en dessous du centile 20 (score «PANSS »< 17), trois sujets entre les centiles 20 et 50 (score $<22$ ), quatre sujets entre les centiles 50 et 90 (score $<30$ ) et un sujet a une symptomatologie négative le situant au centile 90 d'une population de patients souffrant de schizophrénie (score $=30$ ).

Insérer Tableau IV

\section{b) Respect des recommandations}

On observe qu'il existe une recommandation sur quatre qui n'est pas suivie dans la pratique: sur l'ensemble des recommandations, $65 \%$ sont totalement respectées, $10 \%$ partiellement, et $25 \%$ pas du tout. 3 RPC sont plus particulièrement concernées par le non respect ou le respect partiel : la RPC 2 (réponse inadéquate au traitement) qui n'est jamais respectée et les RPC 6 (phase de maintenance) et RPC 7 (posologie) qui sont partiellement respectées. Les RPC 2 et 7 concernent plus de la moitié des patients et totalisent à elles seules dix-huit des vingt-quatre cas de non-respect d'une directive. La recommandation concernant la dépression post-psychotique de la RPC 6 n'est pas respectée pour trois des quatre patients souffrant de ce trouble et les recommandations de cette dernière RPC au sujet du monitoring des patients (contrôles cliniques à effectuer pour le suivi) ne sont que partiellement respectées pour l'ensemble de la population étudiée.

Par contre, les recommandations de la RPC 5 (sélection d'une médication pour éviter des effets secondaires) sont respectées pour huit des douze cas concernés. Plus précisément, les recommandations abordant la prise en charge des dyskinésies tardives (prescription d'un neuroleptique atypique) sont respectées pour les deux patients concernés et pour les huit personnes présentant un syndrome extrapyramidal, les directives sont totalement respectées dans quatre cas, partiellement dans deux, et pas du tout dans deux autres. On constate cependant que malgré le respect de ces recommandations un nombre important de patient présente des effets secondaires

Le tableau $V$ donne la fréquence de pertinence et le degré de respect des RPC de "l'ECGTS " évaluées dans notre étude, en décrivant le nombre de patients concernés pour chaque recommandation.

Insérer Tableau $\mathrm{V}$ 


\section{Discussion}

Même si les RPC de «l'ECGTS » (16) sont en général totalement ou partiellement respectées, la pratique clinique reste perfectible : certaines RPC ne sont que partiellement, voire pas du tout, suivies (et plus de la moitié des patients de l'échantillon présente encore des symptômes psychotiques). Néanmoins, le respect des RPC ne garantit pas forcément la résolution des problèmes cliniques rencontrés : 8 des 12 patients présentant des effets secondaires ont une médication qui respecte les recommandations.

L'échantillon de patients de petite taille ne permet pas de tirer des conclusions statistiques très poussées et l'étude ne tient pas compte des recommandations concernant les traitements non pharmacologiques, qui peuvent influencer la compliance et l'évolution générale de la pathologie et modifier par ce biais le degré de respect des RPC pharmacologiques. Les approches psychosociales et psychothérapeutiques sont cependant plus difficiles à étudier que la pharmacothérapie, notamment parce que les réponses à la psychothérapie ne sont pas toujours aussi rapides et aussi facilement mesurables que les réponses au traitement médicamenteux.

La nature rétrospective de notre étude ne permet pas de mettre en évidence la dynamique du traitement. Les entretiens avec les médecins traitants et la consultation des dossiers médicaux ont certes cherché à repositionner les mesures ponctuelles dans un processus évolutif, mais des études avec des mesures répétées dans le temps, tenant compte de l'évolution de la maladie schizophrénique et de son traitement, sont nécessaires pour saisir avec plus d'acuité le respect de recommandations cliniques.

Enfin, la population étudiée est hétérogène mais des critères d'inclusion large (troubles du spectre de la schizophrénie) ont été délibérément choisis car il nous a semblé plus intéressant d'être au plus près de la réalité de la pratique clinique quotidienne. Par ailleurs, "l'ECGTS» (16) ne donne pas une définition claire du terme « schizophrénie », laissant ainsi une marge d'appréciation concernant le type de patients pour lesquels on peut appliquer les recommandations (d'où l'inclusion des troubles schizotypique et schizo-affectif).

Notre étude montre cependant que le choix et l'application d'une RPC ne sont pas simples. Les RPC actuelles donnent peu ou pas d'instrument de mesure, ni critère précis pour évaluer les problèmes 
cliniques auquel elles font référence. Par exemple, les termes « résistance à un traitement initial » ou "syndrome extra-pyramidal » énoncé dans «l'ECGTS»(16) sont peu précis et ont exigé la recherche d'instruments de mesures cliniques et l'établissement de critères ou valeurs seuils.

Même si certains auteurs reprochent aux RPC (et aux études sur lesquelles elles sont basées) de ne pas intégrer la complexité de la réalité clinique $(20,22)$, elles constituent pourtant une aide à la prise de décision thérapeutique. L'avenir appartient donc à des RPC qui proposent, outre les recommandations cliniques elles-mêmes, les moyens de leur vérification et de leur application sur le terrain.

\section{Bibliographie :}

1. ADDINGTON D, ADDINGTON J, MATICKA-TYNDALE E. Assessing Depression in Schizophrenia : The Calgary Depression Scale. Br J Psychiatry 1993 ; 163 (suppl. 22) : 39-44.

2. AGENCE NATIONALE POUR LE DEVELOPPEMENT DE L'EVALUATION MEDICALE. Stratégies thérapeutiques à long terme dans les psychoses schizophréniques. Texte du consensus. Paris : ANDEM, 1994 : 1-32.

3. AMERICAN PSYCHIATRIC ASSOCIATION. A quick reference guide for treating schizophrenia (based on practice guideline for the treatment of patients with schizophrenia, second edition). www.psych.org (site web de l'APA) 2004: 1-27.

4. BARNES T, R, E. A Rating Scale for Drug-induced Akathisia. Br J Psychiatry 1989; 154 : 672-76.

5. BOCHUD M, VADER JP, BURNAND B. Les recommandations de pratique clinique: des recettes utiles pour le praticien? Med Hyg 1998 ; 56 : 272-78.

6. DAVIS DA, TAYLOR-VAISEY A. Translating guidelines into practice. Can Med Assoc J 1997 ; $157(4): 408-16$.

7. GUELFI JD, LEPINE JP. Positive and Negative Syndrome Scale in L'évaluation clinique standardisée en psychiatrie. Castres, Pierre Fabre ed, 1996 ( tome II ) : 585-91.

8. GUELFI JD, LINGJAERDE O et al. L'échelle d'effets indésirables UKU in L'évaluation clinique standardisée en psychiatrie. Castres, Pierre Fabre ed, 1996 ( tome II ) :651-67.

9. GUELFI JD, WAINTRAUB L. Echelle d'effets secondaires extra-pyramidaux de Simpson et Angus in L'évaluation clinique standardisée en psychiatrie. Castres, Pierre Fabre ed, 1996 (tome II) : 669-76.

10. HAYWARD R. Clinical practice guidelines on trial. Can Med Assoc J 1997 ; 156(12) : 1725-7.

11. KAY SR, FISZBEIN A, OPLER LEWIS A. The positive and negative syndrome scale (PANSS) for schizophrenia. Schizophr Bull $1987 ; 13$ (2): 261-75.

12. LANÇON C, AUQUIER P, REINE $G$ et al. Study of the concurrent validity of the Calgary Depression Scale for Schizophrenics (CDSS). J Affect Disord $2000 ; 58: 107-15$.

13. LEHMAN A F, LIEBERMAN J A et al. Practice guideline for the treatment of patients with schizophrenia in Compendium, American Psychiatric Association practice guidelines for the treatment of psychiatric disorders. Am Psychiatr Assoc ed, 2004 (Virginia) : 249-439. 
14. LEHMAN F, STEINWACHS D M and the co-investigators of the PORT project. Translating research into practice: the Schizophrenia Patient Outcomes Research Team (PORT) treatment recommendations. Schizophr Bull $1998 ; 24$ (1) : 1-10.

15. LEJOYEUX M, GORWOOD P, STALLA - BOURDILLON A et al. Traduction et utilisation de l'échelle de Simpson et Angus de symptômes extra-pyramidaux. Encephale 1993 ; XIX : 1721.

16. McEVOY JP, SCHEIFLER PL, FRANCES A. The Expert Consensus Guidelines Series: Treatment of Schizophrenia. J Clin Psychiatry 1999; 60 (suppl 11) :1-80.

17. MILLER A L, HALL C S, LYNN C M, CHILES J A. The Texas Medication Algorithm Project (TMAP) schizophrenia module. TIMA procedural manual: schizophrenia module 2003. www.dshs.state.tx.us/mhprograms/timasczman.pdf (lien testé le 21.05.06)

18. MILNER $K K_{1}$ VALENSTEIN $M$. A comparaison of guidelines for the treatment of schizophrenia. Psychiatr Serv $2002 ; 53$ (7): 888-90.

19. ORGANISATION MONDIALE DE LA SANTE, PULL $C$ B et al. Schizophrénie, trouble schizotypique et troubles délirants in Classification Internationale des Troubles Mentaux et des Troubles du Comportement (chapitre V). CIM 10/ ICD 10. Masson ed, 1993 : 76-87 et 9497.

20. PILET F. Patient based medicine: une évidence! Med Hyg 2004 ; 2506 : 2354-58.

21. SCHOOLER NR, KANE JM. Research diagnoses for tardive dyskinesia. Arch Gen Psychiatry $1982 ; 39: 486-87$.

22. WASSERFALLEN JB. L'allocation des ressources de santé devrait-elle être evidence-based ? Med Hyg 2004; 2506 : 2367-71. 
Tableau I: Comparaison des Recommandations de Pratique Clinique (RPC) sur le traitement des schizophrénies selon les critères de sélection de Bochud et al. (1998) (5), et Milner \& Valenstein (18).

\begin{tabular}{|c|c|c|c|c|c|c|}
\hline & $\begin{array}{l}\text { "APA Practice } \\
\text { Guideline » (13) }\end{array}$ & $\begin{array}{l}\text { "APA guide rapide } \\
\text { de référence » (3) }\end{array}$ & $\begin{array}{l}\text { Recommandations } \\
\text { «PORT» (14) }\end{array}$ & $\begin{array}{l}\text { "Expert } \\
\text { Consensus } \\
\text { Guideline » (16) }\end{array}$ & $\begin{array}{l}\text { "Algorithmes } \\
\text { TMAP »(17) }\end{array}$ & $\begin{array}{l}\text { Recommandations } \\
\text { françaises } \\
\text { «ANDEM» (2) }\end{array}$ \\
\hline Date Publication & $2004^{1}$ & $2004^{1}$ & $1998^{3}$ & 1999 & 2003 & 1994 \\
\hline Validité Scientifique $^{2}$ & Bonne & Bonne & Bonne & Moyenne & Médiocre & Moyenne \\
\hline Phase de la maladie & $\begin{array}{l}\text { Aiguë et } \\
\text { maintenance }\end{array}$ & $\begin{array}{l}\text { Aiguë et } \\
\text { maintenance }\end{array}$ & $\begin{array}{l}\text { Aiguë et } \\
\text { maintenance }\end{array}$ & $\begin{array}{l}\text { Aiguë et } \\
\text { maintenance }\end{array}$ & Aiguë & Maintenance \\
\hline Comorbidités abordées & Nombreuses & Quelques-unes & Quelques-unes & Quelques-unes & Quelques-unes & Quelques-unes \\
\hline $\begin{array}{l}\text { Variétés des modalités } \\
\text { de traitement }\end{array}$ & Nombreuses & Nombreuses & Nombreuses & Nombreuses & $\begin{array}{l}\text { Pharmacothérapie } \\
\text { uniquement }\end{array}$ & Nombreuses \\
\hline $\begin{array}{l}\text { Nombre total des } \\
\text { recommandations }\end{array}$ & Environ 100 & Environ 100 & 30 & plus de 300 & Environ 40 & Environ 65 \\
\hline $\begin{array}{l}\text { Nombre total des } \\
\text { recommandations } \\
\text { pharmacologiques }\end{array}$ & Environ 45 & Environ 45 & 18 & Environ 43 & Environ 30 & Environ 29 \\
\hline Forme de présentation & $\begin{array}{l}\text { Texte } \\
\text { essentiellement }\end{array}$ & $\begin{array}{l}\text { Algorithmes et } \\
\text { recommandations }\end{array}$ & $\begin{array}{l}\text { Recommandations } \\
\text { brèves (avec niveau } \\
\text { d'évidence précisé) }\end{array}$ & $\begin{array}{l}\text { Recommandations } \\
\text { (sous forme de } \\
\text { tableaux) }\end{array}$ & Algorithmes & Texte \\
\hline Facilité d'emploi & Médiocre & Bonne & Moyenne & Moyenne & Bonne & Médiocre \\
\hline
\end{tabular}

1. Basée sur une revue de la littérature s'arrêtant en 2002

2. Selon Milner \& Valenstein (18)

${ }^{3}$ Basée sur une revue de la littérature s'arrêtant en 1995

(«APA= American psychiatric association », «T-MAP= Texas Medical Algorithm Project », «PORT= The Schizophrenia Patient Outcomes Research Team », «ANDEM= Agence Nationale pour le Développement de l'Evaluation Médicale $\gg$ ) 
Tableau III : Utilisation des Recommandations de Pratique Clinique (RPC) de «l'ECGTS» (16) ${ }^{1}$.

\begin{tabular}{|c|c|c|}
\hline Echelles utilisées dans l'étude ${ }^{2}$ & Valeurs seuil pour chaque échelle considérée $e^{3}$ & $\begin{array}{c}\text { Recommandations de }\left\langle I^{9} \mathbb{E} C \mathrm{TTS}\right\rangle^{4} \\
\text { concernées par le score } \\
\text { problématique }\end{array}$ \\
\hline «UKU » (8) & $\begin{array}{c}\text { score total de } \geq 3 \text { dans } 1 \text { des } 4 \text { catégories générales } \\
\text { et évaluation globale du médecin } \geq 2 \\
\text { ou } \\
\text { score } \geq 1 \text { dans une des } 4 \text { catégories générales } \\
\text { et évaluation globale du patient } \geq 2\end{array}$ & $\operatorname{RPC} 4, \operatorname{RPC} 5, \operatorname{RPC} 8$ * \\
\hline « Calgary depression scale » $(1,12)$ & score de 6 et plus & $\operatorname{RPC} 4, \operatorname{RPC} 6$ * \\
\hline «Simpson et Angus scale » $(9,15)$ & score global $\geq 3$ & $\mathrm{RPC} 5, \mathrm{RPC} 8^{*}$ \\
\hline «AIMS » (21) & $\begin{array}{c}\text { score des mouvements involontaires } \geq 3 \text { dans au moins } 2 \text { parties du corps } \\
\text { ou } \\
\text { score des mouvements involontaires } \geq 4 \text { dans au moins une partie du corps }\end{array}$ & $\operatorname{RPC} 5, \operatorname{RPC} 6, \operatorname{RPC} 8$ * \\
\hline $\begin{array}{l}\text { «Barnes akathisia rating scale » } \\
(4)\end{array}$ & score $\geq 2$ sur l'échelle d'évaluation globale ( $4^{\mathrm{eme}}$ rubrique de l'échelle) & $\operatorname{RPC} 5, \operatorname{RPC} 8^{*}$ \\
\hline «PANSS » $(7,11)$ & $\begin{array}{c}\text { score total } \geq 23 \text { dans une des } 2 \text { échelles (positive - négative) } \\
\text { ou } \\
2 \text { scores } \geq 4 \text { ou un score } \geq 5 \text { dans l'une des } 2 \text { échelles (positive - négative) }\end{array}$ & $\operatorname{RPC} 2, \operatorname{RPC} 4, \operatorname{RPC} 8$ * \\
\hline
\end{tabular}

\footnotetext{
${ }^{1}$ Pour chaque échelle, le score mentionné constitue une indication pour le clinicien qui devra alors vérifier si les RPC indiquées dans la $3^{\text {ème }}$ colonne sont respectées

2 «PANSS = Positive and Negative Syndrome Scale»; "UKU = échelle d'évaluation UKU des effets secondaires »; "AIMS = Abnormal involuntary movement scale »

${ }^{3}$ valeurs au-delà desquelles on considère qu'il y a un problème clinique

4 "ECGTS = Expert consensus guideline for the treatment of schizophrenia »

* cf Tableau II qui décrit les problèmes cliniques abordés par les RPC de "l'ECGTS"
} 
Tableau IV: Principaux problèmes cliniques (effets secondaires, sévérité des symptômes psychotique) pour les 20 patients de l'étude en regard de leur diagnostic et de leur médication psychotrope.

\begin{tabular}{|c|c|c|c|c|c|}
\hline No & $\begin{array}{c}\text { Diagnostics } \\
\text { (selon CM-10) }\end{array}$ & $\begin{array}{l}\text { Score " PANSS " } \\
\text { positive / négative \# }\end{array}$ & $\begin{array}{c}\text { Médication } \\
\text { neuroleptique }\end{array}$ & $\begin{array}{c}\text { Autre médication } \\
\text { psychotrope }\end{array}$ & Effets secondaires \\
\hline
\end{tabular}

$1 \quad F 20.04 ; F 32.10$

$2 \quad F 20.00 ; F 11.22$

F12.25

$3 \quad F 20.1 ; F 42.1 ; F 81.9$

$18 / 22$

F20.3

$5 \quad F 21 ; F 45.4 ; F 10.20$

$15 / 30$

$6 \quad$ F21; F32.11

F20.3

F25.2

$9 \quad F 25.9 ; F 60.2 ; F 19.1$

$10 / 20$

10

F20.15

11

$F 20.14 ; F 70$

F20.3

13 F20.0; F32.10, G47.4

$14 / 7$

14

15

16

17

$F 20.0 ; F 42.2$

$18 / 14$

18

19

20

$10 / 16$

$27 / 26$ olanzapine $15 \mathrm{mg} / \mathrm{j}$

clozapine $100 \mathrm{mg} / \mathrm{j}$

quetiapine $400 \mathrm{mg} / \mathrm{f}$

olanzapine $15 \mathrm{mg} / \mathrm{j}$

risperidone $4 \mathrm{mg} / \mathrm{j}$; chlorprothixène 50 $\mathrm{mg} / \mathrm{j}+45 \mathrm{mg} / \mathrm{j} R$

olanzapine $20 \mathrm{mg} / \mathrm{s}$

olanzapine $30 \mathrm{mg} /$; lévomépromazine 25 $\mathrm{mg} / \mathrm{s}$

olanzapine $20 \mathrm{mg} / \mathrm{j}$; décanoate d'halopéridol $150 \mathrm{mg} / 3 \mathrm{sem}$

clozapine $350 \mathrm{mg} / \mathrm{j}$

thioridazine $60 \mathrm{mg} /$ /

flupentixol dépôt $30 \mathrm{mg} / 3 \mathrm{sem}$

clozapine $175 \mathrm{mg} / \mathrm{j}$

zuclopenthixol dépôt $300 \mathrm{mg} / 3 \mathrm{sem}$

flupentixol dépôt 20 $\mathrm{mg} / 3 \mathrm{sem}$

clozapine $375 \mathrm{mg} / \mathrm{j}$

quetiapine $400 \mathrm{mg} / \mathrm{j}$

sertraline $50 \mathrm{mg} / \mathrm{f}$

clorazepate $10 \mathrm{mg} / \mathrm{j}$ topiramate $200 \mathrm{mg} / \mathrm{j}$

lorazepam 2,5 mg/j R ; clorazepate $10 \mathrm{mg} / \mathrm{j}$

lorazepam $2 \mathrm{mg} / \mathrm{s}$

zolpidem $10 \mathrm{mg} / \mathrm{j} R$

buspirone $30 \mathrm{mg} / \mathrm{s}$

lorazepam $1 \mathrm{mg} / \mathrm{j}$ R;

zolpidem $10 \mathrm{mg} / \mathrm{j}$

procyclidine $10 \mathrm{mg} / \mathrm{j} \mathbf{R}$;

zolpidem $10 \mathrm{mg} / \mathrm{j}$

venlafaxine $300 \mathrm{mg} / \mathrm{j}$

procyclidine $10 \mathrm{mg} / \mathrm{j}$

procyclidine $15 \mathrm{mg} / \mathrm{j} \mathbf{R}$ dyskinésie tardive

difficultés de concentration asthénie; fatigue; hypokinésie, hyposialorrhée

trouble de l'activité onirique hypersialorrhée, vertiges orthostatiques; photosensibilité, troubles.sexuels, syndrome extrapyramidal

syndrome extra-pyramidal

syndrome extra-pyramidal

sulfate de lithium $1320 \mathrm{mg} / \mathrm{j}$;

sulfate de lithium $990 \mathrm{mg} / \mathrm{ji}_{\text {; }}$

hypokinésie, dystonie; troubles de l'accommodation; hyposialorrhée, aménorrhée

indifférence émotionnelle, hypersialorrhée; syndrome extra-pyramidal palpitations, tachycardie, photosensibilité; troubles sexuels.

syndrome extra-pyramidal

syndrome extra-pyramidal; akathisie; polyurie, prise de poids, troubles sexuels. 
Tableau V: Respect et pertinence des Recommandations de Pratique Clinique (RPC) de «The Expert Consensus Guideline for the treatment of schizophrenia " (16) pour les 20 patients de l'étude.

\begin{tabular}{l|c|c|c|c|}
\cline { 2 - 5 } & \multicolumn{3}{|c|}{ Nombre de patients pour lesquels les RPC sont : } \\
& non respectées & $\begin{array}{c}\text { partiellement } \\
\text { respectées }\end{array}$ & $\begin{array}{c}\text { entièrement } \\
\text { respectées }\end{array}$ & non pertinentes \\
\hline RPC 2 & 11 & 0 & 0 & 9 \\
RPC 3 A & 0 & 0 & 5 & 15 \\
RPC 3 B & 0 & 0 & 8 & 12 \\
RPC 4 & 2 & 2 & 14 & 2 \\
RPC 5 & 2 & 2 & 8 & 8 \\
RPC 6 & 0 & 6 & 14 & 0 \\
RPC 7 & 8 & 1 & 7 & 4 \\
RPC 8 A & 1 & 0 & 7 & 12 \\
\hline
\end{tabular}

